\title{
Agua y ciencia ciudadana: vía para la construcción conjunta de soluciones
}

\section{locales}

La ciencia ciudadana representa un nuevo

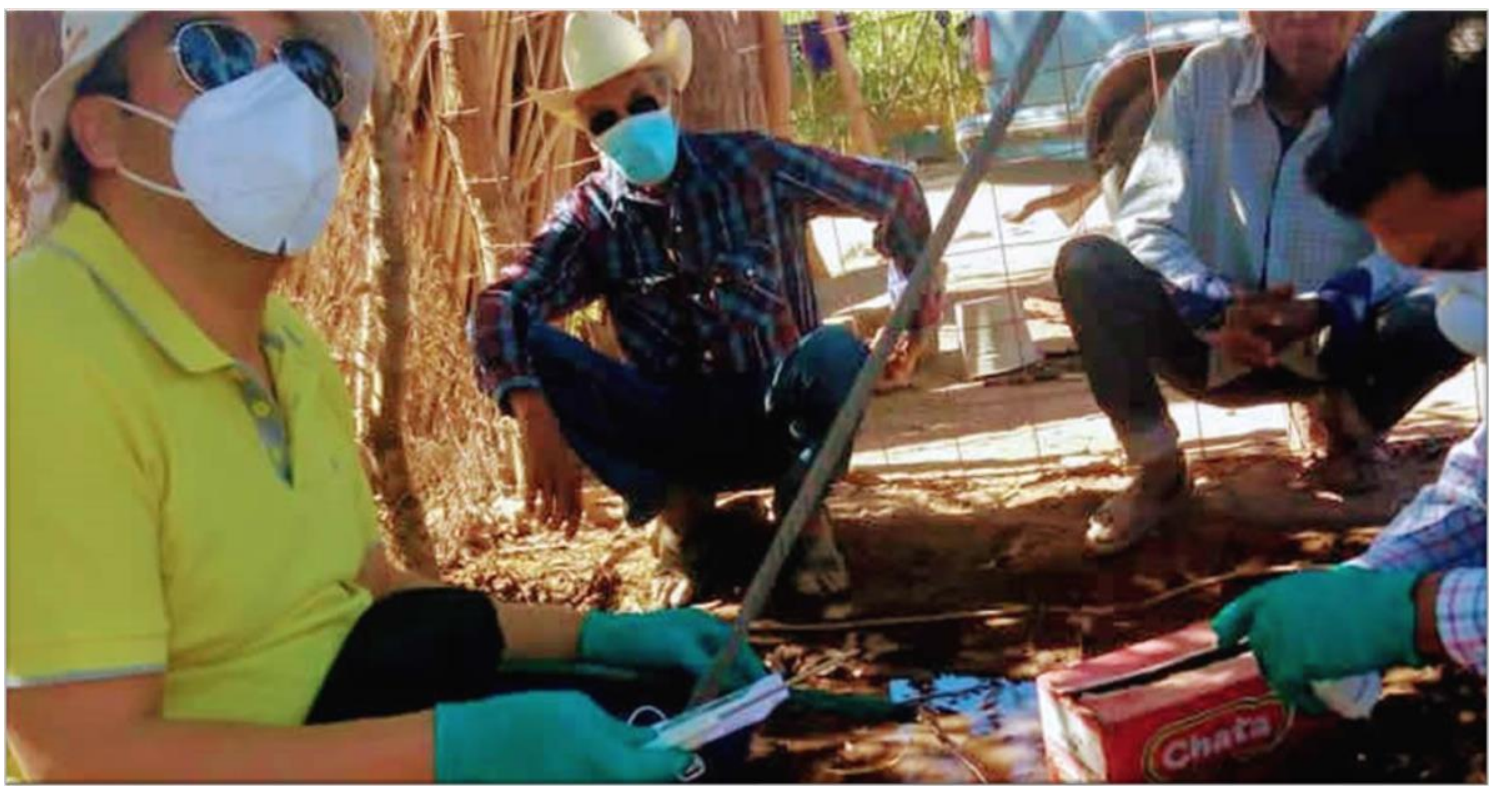
hacer científico.

En todo el mundo se aprecia una clara proliferación del uso de la ciencia ciudadana en temas relativos al agua.

lo que ha permitido el involucramiento de la sociedad en el monitoreo de variables hidrológicas, climáticas y de calidad, y en ejercicios de mapeo y modelado de cuencas hidrológicas.

La ciencia ciudadana representa un nuevo paradigma para el quehacer científico, en el que las personas que viven en un territorio que es objeto de estudio se involucran en las actividades de recolección y análisis de datos, y diseminación de los resultados. Los orígenes de la ciencia ciudadana son también los orígenes de la ciencia moderna; es decir, antes de la profesionalización de la ciencia, los grandes experimentos y aventuras científicas eran realizadas por personas curiosas, cuyo intelecto los llevó a descubrir a partir de la experiencia. Ejemplos de este tipo de personajes son Benjamín Franklin y Charles Darwin (Miller-Rushing et al., 2012).

El florecimiento de la ciencia ciudadana en estudios ambientales se debe a diversos factores, pero quizá el que valga la pena resaltar es el de las crecientes y justas preocupaciones de la sociedad sobre la salud 
PERSPECTIVAS IMTA (0)

$N^{\circ} .30,2020$

MEDIO AMBIENTE

Autor: Adrián Pedrozo Acuña

DOI: $\underline{\text { doi.org/10.24850/b-imta-perspectivas-2020-30 }}$

de su ambiente local y los impactos sobre su propia salud personal y familiar (Carlson y Cohen, 2018; Haklay, 2013).

La aplicación de la ciencia ciudadana en temas relacionados con el al agua es relativamente reciente y está en crecimiento (Njue et al., 2019; Zheng et al., 2018). Existe un gran número de publicaciones que reportan que además de los beneficios científicos, la incorporación de la sociedad en actividades de monitoreo y seguimiento científico tiene el potencial de traer consigo muchos beneficios locales a las comunidades que se involucran en estas actividades (p.ej., Buytaert et al., 2014; McKinley et al., 2015; Thornhill et al., 2019). Entre estos beneficios se encuentran la educación y la cultura ambiental de las personas, su empoderamiento y la construcción de confianza entre las comunidades y las instituciones encargadas de las actividades científicas. Esto resulta en un nuevo paradigma de construcción conjunta de soluciones con base en evidencia científica, pero acompañadas del conocimiento empírico y de la preocupación legítima de las personas que viven en una comunidad, cuyo entorno es objeto de estudio. Los esfuerzos que incorporan la ciencia ciudadana varían en un amplio rango de escalas geográficas y de número de voluntarios, desde estudios a escala local, que involucran un solo voluntario (Walker et al., 2019), hasta estudios globales que incorporan la participación de decenas de miles de voluntarios con datos de estaciones meteorológicas instaladas en hogares y que contribuyen a los conjuntos de datos globales de variables meteorológicas (WOW, 2019).

La mayor parte de las tipologías de ciencia ciudadana relacionadas con el agua incorporan proyectos relacionados con la salud de ecosistemas acuáticos iniciados por residentes locales (Bonney et al., 2009) que se dan a través de la cocreación de proyectos colaborativos que incorporan niveles decrecientes de control ciudadano sobre lo que se monitorea, dónde y cómo se mide, quién conduce el análisis y quién usa los datos. Esto redunda en una forma relevante, pero pasiva, de incorporación de la sociedad a estudios científicos.

Por esta razón, cuando consideramos los beneficios de incorporar la ciencia ciudadana, lo mejor es preguntarse cómo pueden los beneficios de una intervención científica contribuir a mejorar las condiciones de vida de las comunidades. Es decir, cómo el conocimiento adicional que se aporta desde la ciencia y la técnica del agua sirve y se aplica para resolver las preocupaciones locales de las personas, de tal suerte que su bienestar y la salud de sus ecosistemas es mejorado por la intervención de la ciencia. La mayoría de los estudios que incorporan el uso de ciencia ciudadana en temas relacionados con el agua indican beneficios potenciales de su utilización, pero señalan una falta de seguimiento de esos beneficios en el tiempo. Entre los más claramente identificados se encuentran los siguientes:

Compromiso público., Este representa la necesidad de incrementar la conciencia de las personas sobre los problemas ambientales, la democratización de la ciencia y la reconstrucción de confianza entre ciencia y sociedad. (Groffman et al., 2010).

Una mayor conciencia: En estos casos, la participación de las personas en la ciencia y en un proyecto científico incrementa la conciencia sobre el tema en estudio, lo que produce un aprendizaje adicional que resulta de ese involucramiento (Brossard et al., 2005). Este tipo de beneficio está relacionado con actividades de custodia por parte de las personas en sus territorios, lo que resulta en la generación de guardianes del ambiente mejor capacitados y con mayor conocimiento.

Democratización de la ciencia: Este beneficio indica la oportunidad de participación de las personas en la generación de conocimiento científico. Se refiere particularmente a la eliminación de barreras entre los Ilamados técnicos o expertos y el público en general (Freitag y Pfeffer, 2013). 
Generación de confianza entre personas, científicos y autoridades. La construcción de estrategias de atención a problemas hídricos con base en evidencia es crucial. Esto indica la necesidad de involucrar a científicos y técnicos para avanzar en las soluciones definitivas a los problemas hídricos locales. Sin embargo, para que las soluciones sean implementadas de forma exitosa, debe primero generarse un puente de confianza entre las personas que habitan y viven la problemática y los científicos que la atienden. De esta forma se podrá tender un puente de confianza ciudadana hacia las autoridades encargadas de hacer de la solución una realidad (Groffman et al., 2010)

Empoderamiento. El aprendizaje habilita a los ciudadanos involucrados a ganar espacios de participación cívica en los debates de política pública relevantes y en los procesos de toma de decisiones (Turrini et al. 2018).

Cambio en el comportamiento. Resultado de un mayor conocimiento y conciencia sobre una problemática hídrica, se genera un cambio en el comportamiento hacia la conservación y cuidado del agua, en particular hacia un uso sustentable del vital líquido (Jordan et al., 2011).

De esta manera, la ciencia ciudadana tiene un gran potencial para fomentar la mejora del medio ambiente de la mano de las comunidades. Esto, a su vez, redunda en un mejoramiento de las condiciones de salud de las personas que habitan los territorios en los que se identifican problemas y se activan intervenciones técnicas y gubernamentales. Por esta razón, la construcción de una ciencia ciudadana sobre los problemas hídricos nacionales tiene el potencial-como lo ha dicho la Secretaria de Medio Ambiente y Recursos Naturales, María Luisa Albores González- de abrir una gran avenida hacia el bienestar de todas y todos los mexicanos. La ciencia ciudadana en temas de agua nos permitirá cuidar la salud ambiental y la salud de las personas.

Por otro lado, es necesario reconocer que exiten también posibles impactos negativos de la ciencia ciudadana, sobre todo si su implementación no se lleva a cabo con cuidado. Algunos aspectos en los que debemos poner atención cuando se implementan acciones de ciencia ciudadana son los siguientes:

Sobrecarga de las personas. La participación ciudadana en la ciencia, especialmente de comunidades con condiciones económicas difíciles en actividades de monitoreo que se les delegan, tiene el potencial de añadir una carga adicional a las personas, cuyas vidas están ya de por sí estresadas por sus desfavorables condiciones económicas.

Decremento de la confianza. Si la actividad de involucramiento de la sociedad está atada a un financiamiento temporal, es probable que la intervención científica de técnicos, soportada por el proyecto, resulte en un sentimiento de abandono al término del mismo, ya que, por lo general, terminado el proyecto terminan las visitas, y con ello se puede generar decepción y una pérdida de confianza de las comunidades que luego resulte complicado restaurar.

Exclusión. Los procesos participativos requieren una amplia diversidad de ciudadanos y opiniones que permitan construir la necesaria legitimidad de las intervenciones de un proyecto científico. La marginalización de ciertos grupos puede añadir mayor presión y sentimientos de exclusión entre las personas, lo que complica la generación e implementación de soluciones técnicas.

Creación de conflictos. Los conflictos pueden resultar por la falta de confianza de ciertos grupos sociales en datos científicos que no representan sus intereses u opiniones (Baalbaki et al., 2019). Por esta razón, es muy importante el proceso de construcción conjunta y representativo a nivel comunidad. Que nadie se sienta excluido y que nadie que quiera participar se quede atrás. 
PERSPECTIVAS IMTA

$\mathrm{N}^{\circ} .30,2020$

El tener identificados los posibles impactos negativos nos permite diseñar la participación ciudadana en la ciencia con mayor cuidado. En todo el mundo, en lo que se refiere a temas relacionados con el agua, la ciencia ciudadana avanza a pasos agigantados. Hacer del agua el elemento que habilite desarrollo económico equitativo pasa necesariamente por el involucramiento de las personas que habitan las cuencas y que viven de ríos y acuíferos.

Actualmente, el Gobierno de México en su conjunto avanza en la creación de soluciones conjuntamente con las personas. El Instituto Mexicano de Tecnología del Agua acompaña a la Secretaría de Medio Ambiente y Recursos Naturales en la atención de problemas hídricos locales en todo el territorio nacional, donde las comunidades expresan sus preocupaciones legítimas sobre la calidad y cantidad del agua de cuencas y acuíferos. Lo hacemos por que es lo correcto, y por esta razón no es gratuito que nuestros principios rectores sean la ética hídrica, la generación de evidencia científica para las decisiones y la transparencia de los datos y la información que proporcionamos a la sociedad.

\section{Referencias:}

Baalbaki, R., Ahmad, S. H., Kays, W., Talhouk, S. N., Saliba, N. A., \& Al-Hindi, M. (2019). Citizen science in Lebanon - A case study for groundwater quality monitoring. Royal Society Open Science, 6(2),

\section{1. https://doi.org/10.1098/rsos.181871}

Bonney, R., Ballard, H., Jordan, R., McCallie, E., Phillips, T., Shirk, J., \& Wilderman, C. C. (2009). Public participation in scientific research: Defining the field and assessing its potential for informal science education. A CAISE Inquiry

Group Report. Online submission.

Buytaert, W., Zulkafli, Z., Grainger, S., Acosta, L., Bastiaensen, J., De Bièvre, B., ... Zhumanova, M. (2014). Citizen science in hydrology and water resources: Opportunities for knowledge generation, ecosystem service management, and sustainable development. Frontiers in Earth Science, 2(26), 1

\section{1. https://doi.org/10.3389/feart.2014.00026}

Brossard, D., Lewenstein, B., \& Bonney, R. (2005). Scientific knowledge and attitude change: The impact of a citizen science project. International Journal of Science Education, 27(9), 1099- 1121.

Carlson, T., \& Cohen, A. (2018). Linking community-based monitoring to water policy: Perceptions of citizen scientists. Journal of Environmental Management, 219, 168- 177. https://doi.org/10.1016/j.jenvman.2018.04.077 Freitag, A., \&. Pfeffer, M. J. (2013). Process, not product: Investigating recommendations for improving citizen science "success". PLoS One, 8(5), e64079.

Groffman, P. M., Stylinski, C., Nisbet, M. C., Duarte, C. M., Jordan, R., Burgin, A., ... Coloso, J. (2010). Restarting the conversation: Challenges at the interface between ecology and society. Frontiers in Ecology and the Environment. 8(6), 284- 291.

Haklay, M. (2013). Citizen science and volunteered geographic information: Overview and typology of participation. In Crowdsourcing geographic knowledge (pp. 105- 122). Dordrecht: Springer.

Jordan, R., Gray, S., Howe, D., Brooks, W., \& Ehrenfeld, J. (2017). Knowledge gain and behavioral change in citizen science programs. Conservation Biology, 25(6), 1148- 1154. https://doi.org/10.1111/j.1523-1739.2011.01745.x McKinley, D. C., Miller-Rushing, A. J., Ballard, H., Bonney, R., Brown, H., Evans, D. M., ... Ryan, S. F. (2015). Investing in citizen science can improve natural resource management and environmental protection. Issues in Ecology, 2015(19), 1- 27.

Miller-Rushing, A., Primack, R., \& Bonney, R. (2012). The history of public participation in ecological research. Frontiers in Ecology and the Environment, 10(6), 285- 290.

Njue, N., Stenfert Kroese, J., Gräf, J., Jacobs, S. R., Weeser, B., Breuer, L., \& Rufino, M. C. (2019). Citizen science in hydrological monitoring and ecosystem services management: State of the art and future prospects. Science of the Total Environment, 693, 133531. https://doi.org/10.1016/j.scitotenv.2019.07.337

Turrini, T., Dörler, D., Richter, A., Heigl, F., \& Bonn, A. (2018). The threefold potential of environmental citizen science - Generating knowledge, creating learning opportunities and enabling civic participation. Biological Conservation, 225, $176-186$. https://doi.org/10.1016/j.biocon.2018.03.024

Walker, D., Smigaj, M., \& Jovanovic, N. (2019). Ephemeral sand river flow detection using satellite optical remote sensing. Journal of Arid Environments, 168, 17- 25. https://doi.org/10.1016/j.jaridenv.2019.05.006 
PERSPECTIVAS IMTA (0)

$\mathrm{N}^{\circ} .30,2020$

Autor: Adrián Pedrozo Acuña

Wow. (2019). Weather observations website, Exeter: The Met Office. Retrieved

from https://wow.metoffice.gov.uk/

Zheng, F., Tao, R., Maier, H. R., See, L., Savic, D., Zhang, T., ... Popescu, I. (2018). Crowdsourcing methods for data

collection in geophysics: State of the art, issues, and future directions. Reviews of

Geophysics, 56(4), 698- 740. https://doi.org/10.1029/2018rg000616 